\title{
Evaluation of human acellular dermis versus porcine acellular dermis in an in vivo model for incisional hernia repair
}

\author{
Manh-Dan Ngo • Harold M. Aberman • \\ Michael L. Hawes • Bryan Choi • \\ Arthur A. Gertzman
}

Received: 17 January 2011 / Accepted: 16 February 2011/Published online: 6 March 2011

(C) The Author(s) 2011. This article is published with open access at Springerlink.com

\begin{abstract}
Incisional hernias commonly occur following abdominal wall surgery. Human acellular dermal matrices (HADM) are widely used in abdominal wall defect repair. Xenograft acellular dermal matrices, particularly those made from porcine tissues (PADM), have recently experienced increased usage. The purpose of this study was to compare the effectiveness of HADM and PADM in the repair of incisional abdominal wall hernias in a rabbit model. A review from earlier work of differences between human allograft acellular dermal matrices (HADM) and porcine xenograft acellular dermal matrices (PADM) demonstrated significant differences $(P<0.05)$ in mechanical properties: Tensile strength $15.7 \mathrm{MPa}$ vs. 7.7 MPa for HADM and PADM, respectively. Cellular (fibroblast) infiltration was significantly greater for HADM vs. PADM (Armour). The HADM exhibited a more natural, less degraded collagen by electrophoresis as compared to PADM. The rabbit model surgically established an incisional hernia, which was repaired with one of the two acellular dermal matrices 3 weeks after the creation of the abdominal hernia. The animals
\end{abstract}

M.-D. Ngo · B. Choi · A. A. Gertzman $(\bowtie)$

Musculoskeletal Transplant Foundation, Edison, NJ, USA

e-mail: arthur_gertzman@mtf.org

H. M. Aberman

Applied Biological Concepts, Los Alamitos, CA, USA

M. L. Hawes

Charter Preclinical Services, Hudson, MA, USA were euthanized at 4 and 20 weeks and the wounds evaluated. Tissue ingrowth into the implant was significantly faster for the HADM as compared to PADM, 54 vs. $16 \%$ at 4 weeks, and 58 vs. $20 \%$ for HADM and PADM, respectively at 20 weeks. The original, induced hernia defect $\left(6 \mathrm{~cm}^{2}\right)$ was healed to a greater extent for HADM vs. PADM: $2.7 \mathrm{~cm}^{2}$ unremodeled area for PADM vs. $1.0 \mathrm{~cm}^{2}$ for HADM at 20 weeks. The inherent uniformity of tissue ingrowth and remodeling over time was very different for the HADM relative to the PADM. No differences were observed at the 4-week end point. However, the 20 -week data exhibited a statistically different level of variability in the remodeling rate with the mean standard deviation of 0.96 for HADM as contrasted to a mean standard deviation of 2.69 for PADM. This was significant with $P<0.05$ using a one tail $\mathrm{F}$ test for the inherent variability of the standard deviation. No significant differences between the PADM and HADM for adhesion, inflammation, fibrous tissue or neovascularization were noted.

\section{Introduction}

The purpose of this study was to compare the effectiveness of acellular dermal matrices, HADM and PADM, in the repair of incisional abdominal wall hernias in a rabbit model. Incisional hernias commonly occur following abdominal wall surgery. Human acellular dermal matrices (HADM) are widely used in abdominal wall defect repair. 
Xenograft acellular dermal matrices, particularly those made from porcine tissues (PADM), have recently experienced increased usage.

Wide success has been reported for synthetic mesh in the repair of inguinal hernia (Cobb et al. 2005; Novitsky et al. 2007). Abdominal surgery however, can result in an incisional hernia which has a recurrence rate as high as $54 \%$, depending on the techniques used in repair (den Hartog et al. 2008). Primary repair of large abdominal defects have had limited success, with morbidity and recurrence rates of 24 and 18\%, respectively (Bisgaard et al. 2009; Ko et al. 2009).

Prosthetic materials have been used for repair of large abdominal wall defects to provide a scaffold for the reconstruction and reinforcement of the hernia defect. A significant decrease in the recurrence rate was found among patients who had mesh repair when compared with those with suture repair alone (Luijendijk et al. 2000). Equivalent results with onlay and sublay mesh repair techniques were shown (den Hartog et al. 2008).

However, with some biological meshes, problems have been reported with certain meshes that have exhibited degraded biomechanical properties, i.e., the repair site is weaker than if autologous tissue had been used (Collage and Rosengart 2010). As healing and resorption of a biologic scaffold occurs, a loss of tissue support over time may result in the occurrence of abdominal wall laxity or bulging. Foreign body reaction and the potential to act as a nidus for infection, especially in a contaminated environment are risks that have been raised (Engelsman et al. 2007; Bachman and Ramshaw 2008). Furthermore, the potential for harming the viscera underneath due to tissue-biomaterial interactions such as adhesions or erosion is of concern.

Advances in tissue transplant recovery and processing techniques have resulted in the development of acellular dermal sheets from human and animal origin (Ko et al. 2009; Luijendijk et al. 2000; Collage and Rosengart 2010; Engelsman et al. 2007; Bachman and Ramshaw 2008). Human acellular dermal matrices (HADM) have been used for primary hernia repair with good clinical success (Gaertner et al. 2007; Bellows et al. 2007). While the donor source is similar for all of the HADM, differences in recovery and processing techniques can result in different clinical outcomes. Processing of tissue could have profound effects on both handling properties and the in vivo performance of human tissue grafts. Eberli et al. (Eberli et al. 2010) reported delayed cellular infiltration resulting from process-induced differences. It has also been reported (Bachman and Ramshaw 2008) that AlloDerm ${ }^{\circledR}$ human dermal allograft tissue requires pre-stretching prior to implantation. It has further been shown that Flex $\mathrm{HD}^{\circledR}$, a human acellular dermis from the Musculoskeletal Transplant Foundation, possesses greater tensile strength than AlloDerm, the human acellular dermis from LifeCell (MTF data on file 2010). The differences are likely caused by processing-induced degradation. See Fig. 1.

These differences in initial strength and the effect of human versus porcine tissue are to be evaluated to determine the differences in hernia repair rate and the potential for wound failure in the form of laxity or bulging after abdominal hernia repair.

Tissue comparison: human allograft and porcine xenograft

Dermal grafts are incorporated into host tissue by a biological mechanism of enzymatic absorption, neovascularization, and cellular infiltration attendant with collateral, new tissue remodeling. The remodeling step requires the incorporation and proliferation of fibroblast cells into the implanted tissue as it resorbs. A recent study compared human

Tensile Strength of Acellular Dermis (HADM)

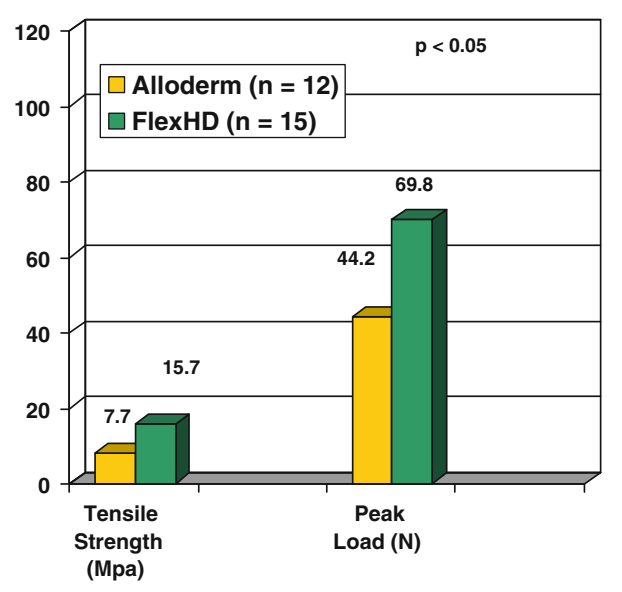

Fig. 1 Tensile strength of acellular dermis (HADM). FlexHD exhibited significantly greater tensile strength than a comparative allograft dermal matrix material, Alloderm 
acellularized dermal matrix with porcine acellularized dermal matrix as a scaffold for human fibroblasts. Fibroblast infiltration, necessary for effective graft healing was reported in a statistically significant greater number in human acellularized dermal matrix than porcine dermal matrix $(83 \%, n=24$ compared with $31 \%, n=49 ; P<0.05$ ) (Badylak 2007).

In a related study (Armour et al. 2006), cells cultured with porcine matrices stack up on the surface of the matrix and became more tightly packed than human matrices after 4 weeks, suggesting a possible delay in fibroblast infiltration. Moreover, in each sample, a significantly greater number of cells were observed below the surface, i.e. within the matrix in human acellularized dermal matrix than in the porcine acellularized dermal matrix $(P<0.05$, Wilcoxin test) at 4 weeks (Table 1).

It is well known that processing procedures could have a profound effect on the biochemistry and properties of collagen-based tissue matrices (Roth et al. 2009; Eberli et al. 2010). Gel electrophoresis (SDS-PAGE) is a well established qualitative technique to detect the quality of the collagen in tissue grafts. (Sykes et al. 1976). In a comparison of PADM to HADM using this characterization method, the quality of the collagen Types I and III present in human ACD was shown to be similar to that of both purified collagen protein and unprocessed skin (Fig. 2). The electrophoresis data does show, however that the HADM samples $(N=5$ lots and 1 replicate per lot) demonstrate a greater quantity of intact alpha collagen chains, the natural collagen protein types present in native dermal tissue. The data suggests a somewhat higher concentration of degraded collagen in PADM as compared to HADM (Fig. 2 ).

Hence, the biochemical results suggest a more natural, less degraded collagen material in human

Table 1 Fibroblast infiltration of porcine and human acellularized dermal matrix at 4 weeks

Cells $^{\mathrm{a}} / 8 \mathrm{~mm}^{\mathrm{m}}$ section $^{\mathrm{b}}$

\begin{tabular}{lr}
\hline HADM & $1072 \pm 80^{\mathrm{c}}$ \\
PADM & $301 \pm 48$ \\
\hline
\end{tabular}

From Armour et al. (2006)

${ }^{\text {a }}$ Cells determined by automated cell counting

b $n=16$ samples, 3 sections per sample

c Significantly different; Wilcoxin signed rank test

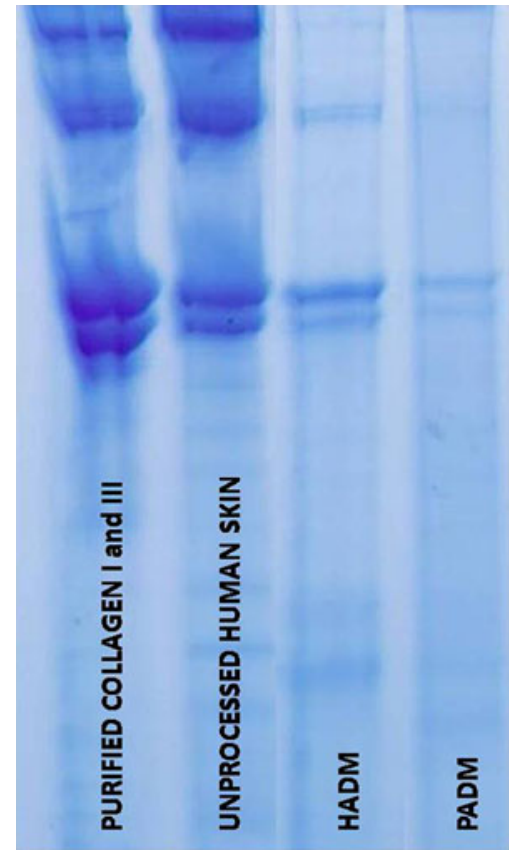

Fig. 2 Comparison of collagen (I, III) quality by gel electrophoresis

dermis as compared to porcine tissue by these biochemical characterization methods.

Reports on the success of HADM have led to the development of alternate sources for the biologic meshes (Jarman-Smith et al. 2004; Catena et al. 2007). Animal derived dermal matrices have thus been developed, principally from porcine sources (Harper 2001; Adedeji et al. 2002; Liyanage et al. 2006). However, there have been few reports on a comparison of HADM and porcine acellular dermal matrices (PADM) in hernia repair (Stanwix et al. 2010). The purpose of this study was to compare the effectiveness of HADM and PADM in a rabbit incisional hernia repair model (Carvalho et al. 2001; LeBlanc et al. 2002; Judge et al. 2007; Da Silva et al. 2009).

\section{Materials and methods}

Sixteen New Zealand white rabbits underwent laparotomy with the creation of an abdominal wall hernia. Three weeks later, hernias were repaired with HADM or PADM. The outcomes, including recurrence, bowel 

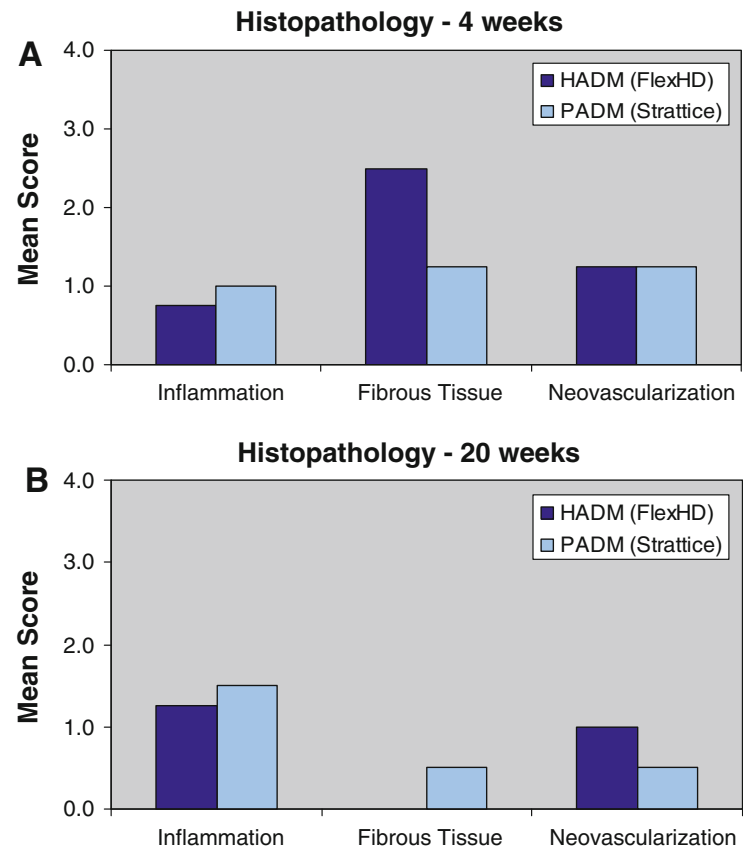

Fig. 3 Histopathological evaluation of the implants. An incisional hernia defect was created in rabbit abdomen. Three weeks following the primary surgery, the defect in each rabbit was repaired with HADM or PADM. Inflammatory response to the implant materials and the growth of fibrous tissue and neovascularization in the implants were evaluated at 4 (A) and 20 (B) weeks later using the following scale: "0" = within normal limits, " $1 "=$ minimal, " $2 "=$ mild, " 3 " = moderate, and " $4 "=$ marked. HADM, human acellular dermal matrices; PADM, porcine acellular dermal matrices

adhesion, histology, degree of remodeling and tensile strength of the explants were evaluated at 4 and 20 weeks after hernia repair (4 animals/ time point). In addition, inflammatory reaction and increases in fibrous tissue ingrowth and neovascularization in the matrices were also examined. This surgical model of inducing an abdominal hernia and then repairing it with the contrasting human and porcine matrices, is intended to closely simulate a real patient condition.

Laboratories and facilities

Biological Test Center, Irvine, CA, USA

Vetpath Services. Mason, OH, USA

Charter Preclinical Services, Hudson, MA, USA

Musculoskeletal Transplant Foundation, Research and Development Laboratories, Edison, NJ, USA
Table 2 Experimental design

\begin{tabular}{lllc}
\hline Group & Biomaterial & 4 weeks & 20 weeks \\
\hline I & HADM & 4 & 4 \\
II & PADM & 4 & 4 \\
& & Total animals & 16 \\
\hline
\end{tabular}

HADM human acellular dermal matrix. Flex $\mathrm{HD}^{\circledR}$ from MTF, Edison, NJ, USA; PADM porcine acellular dermis matrix. Strattice $^{\circledR}$ from LifeNet, Branchburg, NJ, USA

\section{Procedure}

Animal procedures were conducted in accordance with an approved Institutional Animal Care and Use Committee protocol and the Guide for the Care and Use of Laboratory Animals (Euthanasia 2007).

A total of 16 New Zealand White rabbits, weighing 3-4 kg, were randomly assigned to one of the 2 treatment groups: hernia repair with HADM or PADM ( $n=8$ /group). The animals were sacrificed 4 and 20 weeks after hernia repair (see Table 2).

\section{Surgical procedure}

The abdomen, from the xiphoid process to the groin, was surgically prepped. A $10 \mathrm{~cm}$ midline abdominal incision was made from approximately $2 \mathrm{~cm}$ below the xyphoid process to the pubic symphysis. Bilateral skin flaps were raised using blunt dissection of the subcutaneous layer. The rectus abdominis muscle was marked to create a standardized $3 \mathrm{~cm} \times 2 \mathrm{~cm}$ incisional hernia defect lateral to the midline, identified by the linea alba, by excising a full thickness portion of the abdominal wall, including peritoneum, abdominal wall muscle, and fascia, taking care not to injure the underlying viscera. Subcutaneous tissues were then closed using multi-layer suturing with $3 / 0$ Ethilon ${ }^{\circledR}$ (nylon) sutures.

The induced hernia was surgically repaired 3 weeks following the primary surgery. Each animal was prepped for surgery and the original incision beneath the rectus abdominis and above the peritoneal planes, was reopened, taking care not to injure any bowel. The abdomen was re-entered, and the margins of the fascial defect were identified. The size of each defect was recorded followed by an overlay repair with the appropriate implant, ensuring that there was a minimum of $1 \mathrm{~cm}$ margin of overlap on all sides. 
All implants were $5 \mathrm{~cm} \times 6 \mathrm{~cm}$ and prepared according to the manufacturer's directions for use. The PADM were soaked in sterile saline for a minimum of 2 min prior to use, as per manufacturer's instructions. HADM was implanted directly from the package. All implants were held in position with corner and central simple interrupted 3/0 Prolene ${ }^{\circledR}$ polypropylene sutures, followed by a running nonabsorbable suture (3/0 Prolene) around the perimeter of the implant, attaching the dermis as an overlay to the abdominal wall musculature. Subcutaneous tissues and skin were closed using multi-layer suturing with 3/0 Ethilon non-absorbable sutures. Animals were monitored daily to assess general health.

At either 4 or 20 weeks following the repair, 4 animals from each treatment group were humanely euthanized with a barbiturate overdose (Euthanasia 2007). Following euthanasia, lateral and caudal skin incisions were made to expose the surgical site without disruption. The unremodeled portion of the implant was readily discernible by its lack of new vascular or tissue ingrowth. The length and width of the unremodeled zone were then measured in two dimensions using the corner sutures as a guide. Tissue ingrowth was calculated by determining the unremodeled area as the percentage of the original implant $\left(5 \mathrm{~cm} \times 6 \mathrm{~cm}=30 \mathrm{~cm}^{2}\right)$. Any evidence of bowel adhesion, hernia formation, or abdominal bulging was noted: Hernia was defined as macroscopic rupture of the implant in the area of the surgical repair noted at the time of retrieval, but it could also result from graft contracture or suture pull out and visible hernia defect. Bulging was defined as a stretching of the implanted biomaterial due to intraabdominal pressure with ballooning greater than $3 \mathrm{~cm}$ relative to the abdominal wall. The extent of vascularization and tissue ingrowth in the implant was noted.

The abdomen was opened with a lateral incision through the rectus abdominis muscle beneath the xyphoid to prevent perforation of the implanted grafts. Bowel adhesion to the biomaterial was scored for extent $(\%)$ using the Modified Diamond Scale (0; 0\%; 1, <25\%; 2, 25-50\%; and 3, >50\%) (Adedeji et al. 2002). After assessing the levels of adherence, the adhesions were then freed from the implant. The implant area, along with sufficient surrounding tissue was harvested to enable histological and mechanical evaluation.

\section{Histology}

John Roth previously established the host-implant cellular response for HADM in the identical rabbit incisional hernia model (Roth et al. 2009). The analogous cellular response for the PADM from this study is compared to the HADM from Roth in Fig. 4.

In order to determine the nature and extent of incorporation of the implant into the abdominal wall, tissue specimens were taken at the region where the implant and abdominal wall overlapped and from the implant over the defect site and submitted for histological examination using a standard haemotoxylin and eosin staining. Histopathologic evaluation of the local reaction to the implanted surgical mesh was conducted by one of us (HMA) and included assessment of inflammation and vascularization. Microscopic findings were given an inflammatory or adhesion severity score based upon the following scale: "0" = within normal limits, "1" = minimal, $" 2 "=$ mild, " $3 "=$ moderate, and " $4 "=$ marked.

Typical sections from representative explants at 4 and 20 weeks are shown in Fig. 4. Please note that these are histological sections taken from tissue blocks preserved from the Roth (Roth et al. 2009) study and compared, by an independent, blinded

Fig. 4 Representative photomicrographs: see the Raw Data for individual descriptions of samples. The photomicrographs below are meant to be representative of the group data generated for the studies. A Representative photomicrographs of FlexHD and Strattice hernia repair implants at 4 weeks postimplantation. Photos are from the interface between the implant (black arrows) and the host skeletal muscle (blue arrows). Integration of the host tissue and the implant is modest in both groups at this early time point, though the FlexHD appears slightly better. Notice the wide tissue gap (bottom right, black arrow) in the Strattice implant that is lined by a layer of macrophages (yellow arrows) and degenerate cellular debris (blue arrows). While there is also inflammation in the FlexHD sample (top right, black arrows) there is better tissue integration and an infiltration of small capillaries (top right, yellow arrows). Trichrome stain; $40 \times$ and $400 \times$ magnification. B Representative photomicrographs of FlexHD and Strattice hernia repair implants at 20 weeks post-implantation. Photos are from the interface between the implant (black arrows) and the host skeletal muscle (blue arrows). Integration of the host tissue and the implant is good in the FlexHD sample with few tissue gaps and minimal inflammation. Integration of the host tissue and the implant is still minimal in the Strattice implant; notice the tissue gaps (bottom right, blue arrow) and persistence of inflammation (bottom right, black arrows). Trichrome stain; $40 \times$ and $400 \times$ magnification 
A
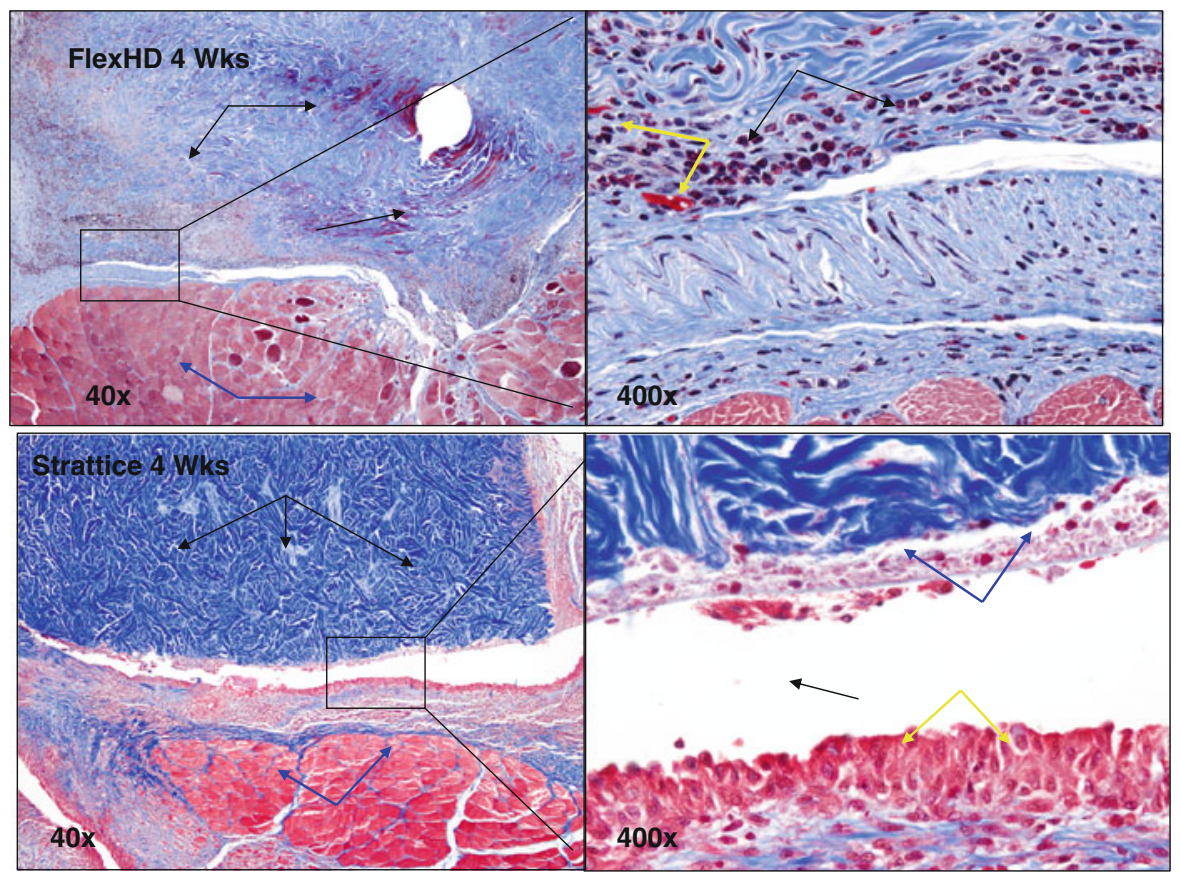

B
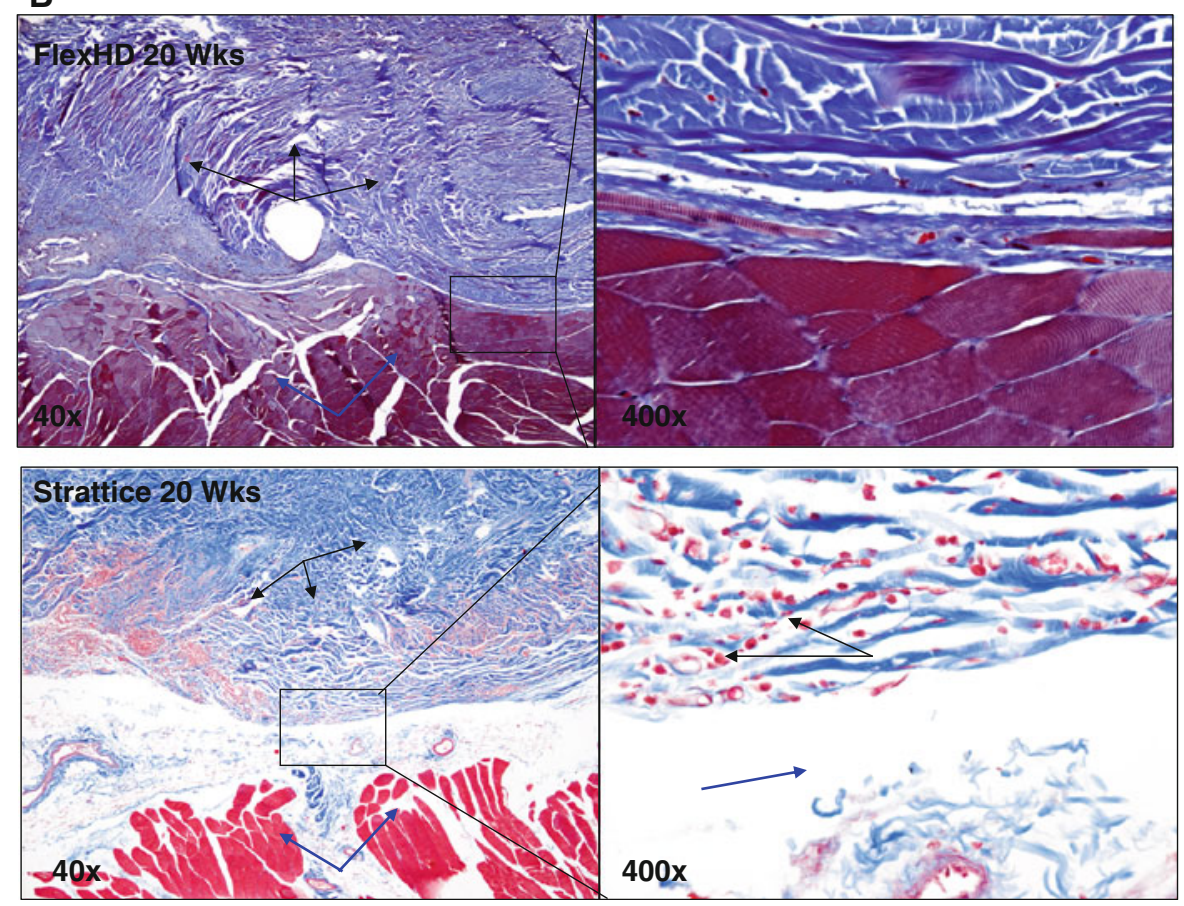
pathologist to sections prepared simultan eously and with the identical procedure from this study.

\section{Mechanical testing}

The tensile properties of the PADM and HADM were measured before implantation on a large, representative sample. Mechanical testing was performed using an MTS 858 Mini Bionix instrument mounted with a $250 \mathrm{~N}$ load cell. Each test sample was prepared from the central-most portion of the explant. Fresh specimens were prepared as dogbone shapes for tensile testing as described in ASTM D638 Type V. The number of dogbones was determined by the available tissue; 1-2 dogbones per explant were obtained.

Test articles were loaded into pneumatic action grips with a gage length of approximately $25 \mathrm{~mm}$ and pulled uniaxially at a strain rate of $50.8 \mathrm{~mm} / \mathrm{min}$ until the mid-section broke. The peak load was measured and recorded, and tensile strength at peak (Ultimate Tensile Strength) was calculated by dividing the peak load by the specimen cross-sectional area.

Sample thickness was measured using a laser micrometer, Bench Mike 4050S Z-Mike by BETA LaserMike (Dayton, OH USA). Three measurements were made on each dog bone sample along the narrow midsection and an average was calculated. The mean thickness was utilized to calculate inherent tensile strength.

Statistical methods included student-t test based on standard deviation. Significant differences were noted for $P<0.05$.

\section{Results}

Gross examination

All sixteen animals recovered uneventfully from both the hernia creation and repair procedures. Following necropsy, gross examination revealed that the HADM implants were well incorporated into the surrounding tissue, exhibiting tissue ingrowth from both the subcutaneous and peritoneal sides. Tissue ingrowth, as expressed as the percentage of area reduction relative to the original $30 \mathrm{~cm}^{2}$ implant area appeared minimal in the PADM implants at 4 weeks after repair. At the 4-week time point, $54 \pm 4.3 \%$ (mean \pm SEM, $n=4)$ of the HADM tissue had incorporated compared to $16 \pm 3.4 \%(n=4 ; P<0.05$ vs. HADM $)$ in the PADM group (Fig. 1). By 20 weeks, 2 out of the 4 PADM implants showed graft thinning and resorption. Of the animals with PADM intact, minimal tissue ingrowth was observed $(19.7 \pm 18.1 \%, n=2)$, while all of the HADM implants were intact at 20 weeks and showed $58 \pm 4.1 \%(n=4 ; P<0.05$ vs. PADM $)$ tissue ingrowth (Fig. 5). These differences, at both 4 and 20 weeks were statistically significant.

The inherent uniformity of tissue ingrowth and remodeling over time was very different for the HADM relative to the PADM. No differences were observed at the 4-week end point. However, the 20-week data exhibited a statistically different level of variability in the rate of remodeling rate with the mean standard deviation of 0.96 for HADM as contrasted to a mean standard deviation of 2.69 for PADM. This was significant with $P<0.05$ using a one tail $\mathrm{F}$ test for the inherent variability of the standard deviation.

The area of hernia defect created initially was $6 \mathrm{~cm}^{2}(3 \mathrm{~cm} \times 2 \mathrm{~cm})$. The size of this defect was decreased in both groups, with the HADM group showing greater reduction compared to PADM at both time points. The differences were not statistically

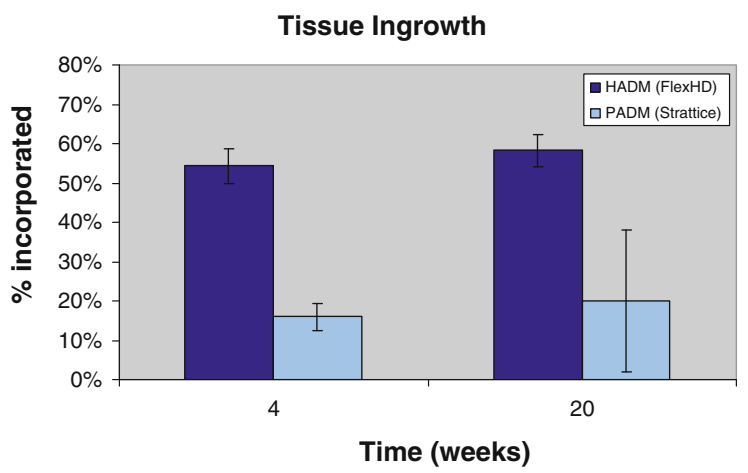

Fig. 5 Tissue ingrowth in the implants. An incisional hernia defect was created in rabbit abdomen. Three weeks following the primary surgery, the defect in each rabbit was repaired with HADM or PADM. Tissue ingrowth in the implants of each group was measured at 4 and 20 weeks later. All values are mean $\pm \operatorname{SEM}(n=4)$, except for PADM at 20 weeks where 2 out of the 4 PADM showed graft thinning and resorption. The results shown with PADM at this time point were from the two intact implants (mean \pm range of error, $n=2$ ). HADM, human acellular dermal matrices; PADM, porcine acellular dermal matrices 
significant. The remaining areas of hernia defect were $2.0 \pm 0.5(n=4)$ and $2.7 \pm 0.9(n=4) \mathrm{cm}^{2}$ in the PADM group when measured at 4 and 20 weeks, respectively, while the respective values in the HADM group were $1.3 \pm 0.4$ and $1.0 \pm 0.2 \mathrm{~cm}^{2}$ $(P>0.05$ vs. PADM) (Fig. 6).

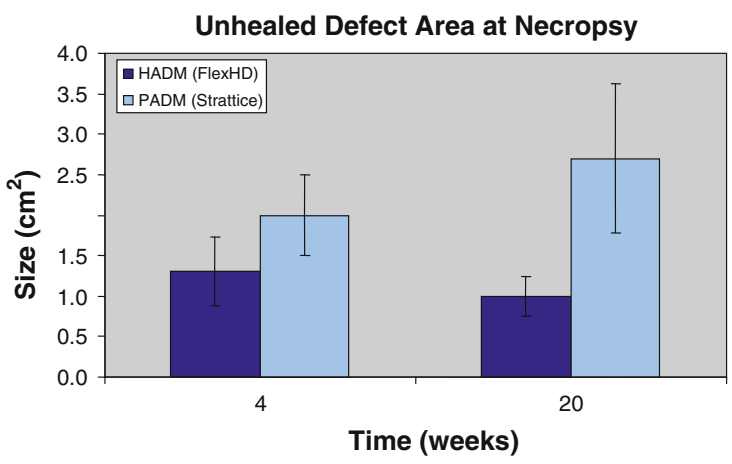

Fig. 6 Area of unhealed defect. A $3 \mathrm{~cm} \times 2 \mathrm{~cm}$ incisional hernia defect was created in rabbit abdomen. Three weeks following the primary surgery, the defect in each rabbit was repaired with HADM or PADM. The unhealed defect size in each group was measured at 4 and 20 weeks later. All values are mean $\pm \operatorname{SEM}(n=4)$. HADM, human acellular dermal matrices; PADM, porcine acellular dermal matrices
Bowel adhesion to the implants was minimal in both groups at 4 weeks; the mean adhesion scores for the HADM and PADM groups were $1.0 \pm 0.0$ $(n=4)$ and $0.8 \pm 0.3$, respectively $(P>0.05)$. They were primarily loose, omental adhesions. Mild omental adhesions were similarly observed in both groups at 20 weeks. No significant differences were noted.

\section{Mechanical strength}

The tensile strengths [presented as mean (standard deviation)] determined for the HADM and PADM controls prior to implantation were 15.7 (5.4) $(n=19)$ and 10.2 (4.3) $(n=5) \mathrm{MPa}$, respectively. In HADM, tensile strengths decreased to $9.0 \pm 1.0$ $(n=4)$ and $3.2 \pm 0.6 \mathrm{MPa}$ after 4 and 20 weeks of implantation, respectively (Table 1). The PADM implants had a tensile strength of $10.8 \pm 1.5$ $(n=4) \mathrm{MPa}$ at 4 weeks. By 20 weeks, apparently due to more rapid graft resorption, only 2 of the 4 the PADM implants had enough material for testing, with an average tensile strength of $0.4 \pm 0.2 \mathrm{MPa}$ (Fig. 7).

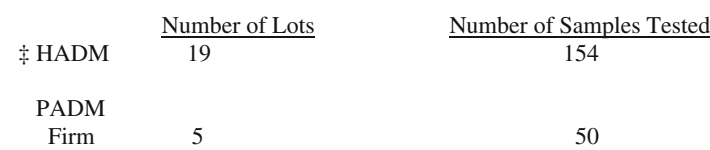

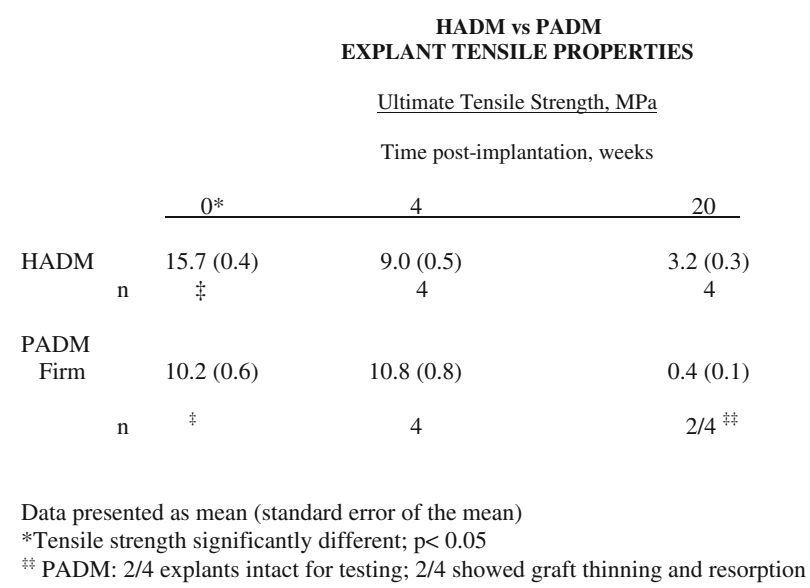

Fig. 7 Tensile strength of explant. An incisional hernia defect was created in the rabbit abdomen. Three weeks following the primary surgery, the defect in each rabbit was repaired with HADM or PADM. Tensile strength of the implants was determined at 4 and 20 weeks later using an MTS 858 Mini Bionix. All values are mean $\pm \operatorname{SEM}(n=4)$. The results shown with PADM at the 20 week time point were from the two intact implants (mean \pm range of error, $n=2$ ). HADM, human acellular dermal matrices; PADM, porcine acellular dermal matrices. The tensile data from the pre-implant phase (time zero) was taken from earlier testing by the identical test protocol and from a larger, multi-donor sample 


\section{Histology}

Histopathology of the implants was assessed microscopically at both time points. At 4 weeks, there were no significant differences in the inflammatory response to the implant materials. In rabbits implanted with HADM and PADM, there was minimal to mild inflammation, fibrosis, and neovascularization of the connective tissues (Fig. 5a). In one animal, mild growth of blood vessels in the HADM was observed, while no formation of blood vessels was noted in the PADM.

At 20 weeks, the inflammatory reaction was similar in both groups. There was also minimal to mild neovascularization of the connective tissues observed in both groups (Fig. 5b). Formation of new blood vessels was seen in one of the HADM grafts In contrast, no blood vessel was formed in the PADM group.

\section{Limitations of the study}

The study results are limited by the small number of animals used for each time point and test material $(n=4)$. The statistical power was therefore constrained. Nevertheless, differences in both the tensile strength and degree of tissue incorporation rose to a level of statistical significance.

Use of the rabbit model created a situation wherein both materials tested were xenogeneic to the rabbit (human and porcine). In actual surgical use, the porcine PADM is xenogeneic to the human patient while the human HADM is allogeneic. This creates a limitation on the relevancy of the model to human use.

Studies with a larger number of animals and for longer observational times than the 20 weeks done here are needed. Clinical trials in humans are also being planned.

\section{Discussion}

This study was intended to evaluate the new porcine xenograft ADMs for abdominal wall repair of incisional hernias. Numerous studies have reported that incisional hernia is a frequent complication following abdominal surgery (Bisgaard et al. 2009; Ko et al. 2009). Controversy as to the best operative repair technique still exists as the recurrence rate may be as high as $54 \%$ (den Hartog et al. 2008). Synthetic prosthetic materials have been shown to decrease recurrence significantly but the risk of prosthetic infection is a concern (Engelsman et al. 2007; Bachman and Ramshaw 2008). Other complications reported in clinical studies with synthetic mesh repair include bowel adhesion and abdominal bulging and contraction (Luijendijk et al. 2000). In the present study, minimal inflammatory reaction with no bowel adhesion or abdominal bulging were similarly found in both the HADM and PADM groups. These results suggest that acellular dermal matrices may be superior to synthetic mesh in this regard.

The advantages and disadvantages for allograft versus xenograft tissue in transplantation have long being debated. Comparison of xenograft and allograft heart valves has shown that there is no mechanism to replace the collagen within xenograft, resulting in a gradual breakdown from proteolysis or mechanical stresses. In this study, a rabbit model of hernia was used to compare the effectiveness of HADM and PADM in defect repair (Carvalho et al. 2001; LeBlanc et al. 2002; Judge et al. 2007; Da Silva et al. 2009). This model has been utilized for evaluating the biocompatibility, integration, and strength of the implants under clinically relevant conditions (Roth et al. 2009; Hilger et al. 2006; Eberli et al. 2010). In this model, both the HADM and PADM are xenografts. Nevertheless, it is interesting to note that $50 \%$ of the PADM showed graft thinning and resorption while all of the HADM were intact at 20 weeks in the present study, suggesting that the difference in the behavior of the two materials could not be attributed to both being xenografts, or that xenograft reaction to the host is greater for the PADM. In addition and as noted earlier, these performance differences could also be the result of a combination of both in processing and the porcine versus human origins of the implanted tissue, per se.

The effectiveness of hernia repair is largely determined by the quality of the repair and time it takes to heal the defect. In the present study, 54\% tissue ingrowth in the HADM was observed by 4 weeks, compared with the $16 \%$ seen in the PADM (Fig. 5). Based on the unremodeled implant area, the HADM remodeled more consistently, particularly at the 20 -week period. Furthermore, the remaining defect size in the HADM was smaller than that of the PADM at the same time point (Table 1). Tensile strengths of HADM and PADM were equivalent at 4 weeks, but the former was almost 8 times stronger than the latter after 20 weeks. These effects may be 
related to the higher rate of fibroblast infiltration for human versus porcine biological matrices as reported by Badylak (Badylak 2007).

\section{Conclusion}

The results reported in the present study showed that in this rabbit model, neither HADM nor PADM was associated with complications such as adverse inflammatory reaction, bowel adhesion, and abdominal bulging, as have been identified in clinical studies using synthetic or other meshes. The HADM, however, appeared to be superior to PADM in terms of initial strength, and the degree and rate of tissue ingrowth/ remodeling. The HADM also showed a more consistent uniform remodeling rate and provided stronger support at the 20 week time point. Clinical studies of both human and porcine biological meshes are in progress.

This study was supported in part by a project grant from the Musculoskeletal Transplant Foundation, Edison, New Jersey, USA.

Open Access This article is distributed under the terms of the Creative Commons Attribution Noncommercial License which permits any noncommercial use, distribution, and reproduction in any medium, provided the original author(s) and source are credited.

\section{References}

Adedeji OA, Bailey CA, Varma JS (2002) Porcine dermal collagen graft in abdominal-wall reconstruction. Br J Plast Surg 55:85-86

Armour AD, Fish JS, Woodhouse KA, Semple JL (2006) A comparison of human and porcine acellularized dermis: interactions with human fibroblasts in vitro. Plastic Reconstr Surg 117(3):845-856

ASTM D 638, ISO 527-1 standard test method for determining tensile properties of plastics

AVMA Guidelines on Euthanasia (2007) American Veterinary Medical Association

Bachman S, Ramshaw B (2008) Prosthetic material in ventral hernia repair: how do I choose? Surg Clin North Am 88(1):101-112 (Ramshaw, B. and S. Bachman (2007). Surgical Materials for Ventral Hernia Repair Biologic Mesh Part 2 of 3. General Surgery News, McMahon Publishing: 1-16.)

Badylak SF (2007) The extracellular matrix as a biologic scaffold material. Biomaterials 28(25):3587-3593

Bellows CF, Albo D, Berger DH, Awad SS (2007) Abdominal wall repair using human acellular dermis. Am J Surg 194:192-198
Bisgaard T, Kehlet H, Bay-Nielsen MB, Iversen MG, Wara P, Rosenberg J, Friss-Andersen HF, Jorgensen LN (2009) Nationwide study of early outcomes after incisional hernia repair. Br J Surg 96:1452-1457

Carvalho ZM, Fagundes DJ, Rodrigues OR, Shimidht Jr A (2001) Incisional hernia: an experimental model in rabbits. Acta Cir Bras [serial on the Internet] 16(2)

Catena F, Ansaloni L, Gazzotti F, Gagliardi S, Di Saverio S, D'Alessandro L L, Pinna AD (2007) Use of porcine dermal collagen graft (Permacol) for hernia repair in contaminated fields. Hernia 11:57-60

Cobb WS, Kercher KW, Heniford BT (2005) The argument for lightweight polypropylene mesh in hernia repair. Surg Innov 12(1):63-69

Collage RD, Rosengart MR (2010) Abdominal wall infections with in situ mesh. Surg Infect (Larchmt) 11(3):311-318

Da Silva W, Fagundes DJ, Seidel AC, Taha MO (2009) Animal model of chronic abdominal hernia in rabbit. Acta Circ Bras 24:256-261

den Hartog D, Dur AH, Tuinebreijer WE, Kreis RW (2008) Open surgical procedures for incisional hernias. Cochrane Database Syst Rev 3: CD006438

Eberli D, Rodriguez S, Atala A, Yoo JJ (2010) In vivo evaluation of acellular human dermis for abdominal wall repair. J Biomed Mater Res A 93:1527-1538

Engelsman AF, van der Mei HC, Ploeg RJ, Busscher HJ (2007) The phenomenon of infection with abdominal wall reconstruction. Biomaterials 28(14):2314-2327

Gaertner WB, Bonsack ME, Delaney JP (2007) Experimental evaluation of four biological prostheses for ventral hernia repair. J Gastointest Surg 11:1275-1285

Harper C (2001) Permacol: clinical experience with a new biomaterial. Hop Med 62:90-95

Hilger WS, Walter A, Zobitz ME, Leslie KO, Magtibay P, Cornella J (2006) Histological and biomechanical evaluation of implanted graft materials in a rabbit vaginal and abdominal model. Am J Obstet Gynecol 195:1826-1831

Jarman-Smith ML, Bodamyali T, Stevens C, Howell JA, Horrucks M, Chaudhuri JB (2004) Porcine collagen crosslinking, degradation and its capability for fibroblast adhesion and proliferation. J Mater Sci Mater Med 15:925-932

Judge TW, Parker DM, Dinsmore RC (2007) Abdominal wall hernia repair: a comparison of sepramesh and parietex composite mesh in a rabbit hernia model. J Am Coll Surg 204:276-281

Ko JH, Salvay DM, Paul BC, Wang EC, Dumanian GA (2009) Soft polypropylene mesh, but not cadaveric dermis, significantly improves outcomes in midline hernia repairs using the components separation technique. Plast Reconstr Surg 124:836-847

LeBlanc KA, Bellanger D, Rhynes KV, Baker DG, Stout RW (2002) Tissue attachment strength of prosthetic meshes used in ventral and incisional hernia repair. A study in the New Zealand White rabbit adhesion model. Surg Endosc 16:1542-1546

Liyanage SH, Purohit GS, Frye JN, Giordano P (2006) Anterior abdominal wall reconstruction with a Permacol implant. J Plast Reconstr Aesthet Surg 59:553-555

Luijendijk RW, Hop WCJ, van den Tol MP, de Lange DCD, Braaksma MMJ, IJzermans JNM, Boelhouwer RU, de Vries BC, Salu MKM, Wereldsma JCJ, Bruijninckx 
CMA, Jeekel J (2000) A comparison of suture repair with mesh repair for incisional hernia. $N$ Engl J Med 343: 392-398

MTF data on file (2010)

Novitsky YW, Harrell AG, Hope WW, Kercher KW, Heniford BT (2007) Meshes in hernia repair. Surg Technol Int 16:123-127

Roth JS, Dexter DD, Lumpkins K, Bochicchio GV (2009) Hydrated vs. freeze-dried human acellular dermal matrix for hernia repair: a comparison in a rabbit model. Hernia 13:201-207

Stanwix MG et al. (2010) Abdominal ventral hernia repair with current biological prostheses. Ann Plast Surg (in press)

Sykes B, Puddle B, Francis M, Smith R (1976) The estimation of two collagens from human dermis by interrupted gel electrophoresis. Biochem Biophys Res Commun 72(4): 1472-1480 